\title{
Quantifying the influences of various ecological factors on land surface temperature of urban forests ${ }^{\text {品 }}$
}

\author{
Yin Ren ${ }^{\text {a, b, * , Lu-Ying Deng }}{ }^{\text {a, c }}$, Shu-Di Zuo ${ }^{\text {a, b }}$, Xiao-Dong Song ${ }^{\text {d }}$, Yi-Lan Liao ${ }^{\text {e, }}$ \\ Cheng-Dong Xu ${ }^{\mathrm{e}}$, Qi Chen ${ }^{\mathrm{f}}$, Li-Zhong Hua ${ }^{\mathrm{g}}$, Zheng-Wei Li ${ }^{\mathrm{h}}$ \\ a Key Laboratory of Urban Environment and Health, Key Laboratory of Urban Metabolism of Xiamen, Institute of Urban Environment, Chinese Academy of \\ Sciences, Xiamen, 361021, China \\ ${ }^{\mathrm{b}}$ Ningbo Urban Environment Observation and Research Station-NUEORS, Chinese Academy of Sciences, Ningbo, 315800, China \\ ${ }^{\mathrm{c}}$ University of Chinese Academy of Sciences, Beijing, 100049, China \\ d College of Environment and Natural Resources, Zhejiang University, Hangzhou, 310058, China \\ e State Key Laboratory of Resources and Environmental Information Systems, Institute of Geographic Sciences and Nature Resources Research, Chinese \\ Academy of Sciences, Beijing, 100101, China \\ ${ }^{\mathrm{f}}$ Department of Geography, University of Hawai'i at Manoa, Honolulu, 96822, USA \\ ${ }^{g}$ Department of Spatial Information Science and Engineering, Xiamen University of Technology, Xiamen, 361024, China \\ h China United Network Communications Group Co. Ltd, Nanjing, 210000, China
}

\section{A R T I C L E I N F O}

\section{Article history:}

Received 14 January 2016

Received in revised form

30 May 2016

Accepted 2 June 2016

Available online 16 June 2016

\section{Keywords:}

Urban forest

Land surface temperature (LST)

Spatial statistical analysis

GeogDetector model

Multiple ecological factors

Driving mechanism

Integrated quantitative analysis

\begin{abstract}
A B S T R A C T
Identifying factors that influence the land surface temperature (LST) of urban forests can help improve simulations and predictions of spatial patterns of urban cool islands. This requires a quantitative analytical method that combines spatial statistical analysis with multi-source observational data. The purpose of this study was to reveal how human activities and ecological factors jointly influence LST in clustering regions (hot or cool spots) of urban forests. Using Xiamen City, China from 1996 to 2006 as a case study, we explored the interactions between human activities and ecological factors, as well as their influences on urban forest LST. Population density was selected as a proxy for human activity. We integrated multi-source data (forest inventory, digital elevation models (DEM), population, and remote sensing imagery) to develop a database on a unified urban scale. The driving mechanism of urban forest LST was revealed through a combination of multi-source spatial data and spatial statistical analysis of clustering regions. The results showed that the main factors contributing to urban forest LST were dominant tree species and elevation. The interactions between human activity and specific ecological factors linearly or nonlinearly increased LST in urban forests. Strong interactions between elevation and dominant species were generally observed and were prevalent in either hot or cold spots areas in different years. In conclusion, quantitative studies based on spatial statistics and GeogDetector models should be conducted in urban areas to reveal interactions between human activities, ecological factors, and LST.
\end{abstract}

(c) 2016 Elsevier Ltd. All rights reserved.

\section{Introduction}

The urban heat island effect caused by rapid urbanization leads to a multitude of eco-environmental problems such as increased energy consumption, photochemicals, smog, slow-diffusing

\footnotetext{
This paper has been recommended for acceptance by Chen Da.

* Corresponding author. Key Laboratory of Urban Environment and Health, Key Laboratory of Urban Metabolism of Xiamen, Institute of Urban Environment, Chinese Academy of Sciences, Xiamen, 361021, China.

E-mail address: yren@iue.ac.cn (Y. Ren).
}

pollutants, and human respiratory disease (Buyantuyev and $\mathrm{Wu}$, 2010). Urban forests are the main source of natural productivity in urban areas (Salvati et al., 2014; Cetin, 2015). The cooling effect (cold island effect) caused by dense shade from branches and leaves limits direct radiant heat from the sun and reflected heat from pavement, walls, and adjacent objects; water transpiration through leaves further consumes radiant heat. The cooling effect plays an extremely important role in mitigating the urban heat island effect, adjusting urban climate, and addressing future climate change (Kong et al., 2014).

Many studies have confirmed an obvious cooling effect in the 
land surface temperature (LST) of urban forests compared with surrounding areas, and this effect is closely related to the type (e.g., tree species, forest age, forest canopy) and spatial characteristics (e.g., shape, area) of urban forests. Many scholars have applied field survey data or remote sensing images of various resolutions (Kuang et al., 2014; Ren et al., 2014; Zhao et al., 2016b) and adopted multivariate statistical analyses to study the relationship between urban forest LST and ecological factors such as forest area, canopy density, vegetation richness, and vegetation index (Chen et al., 2006). However, most of these previous studies analyzed the relationship between urban forest LST and a single ecological factor without comprehensively considering the quantitative relationships between LST and multiple ecological factors (Heffernan et al., 2014; Zhao et al., 2016a). During the interactions between human and natural factors, the diversity, complexity, nonlinearity, and heterogeneity of various ecological factors jointly influence the entire process of urban forest growth. Variations in the intensity of ecological factors, including biotic (e.g., area, dominant tree species, forest age, canopy density) and abiotic factors (e.g., topography, soil), result in a high degree of spatial and temporal heterogeneity in urban forest LST (Shoshany et al., 2005). The effects of human activities further enhance spatial and temporal heterogeneity (Bump et al., 2009). However, few reports have clarified the effects (including strengths and directions) of interactions between human activities, multiple ecological factors, and urban forest LST or quantified the spatial heterogeneity of urban forest LST. The influence of the above-mentioned factors on urban forest LST at the macro-scale (e.g., regional, national, or global) and the strength of interactions between these factors remain unclear (Buyantuyev and Wu, 2010).

Quantifying the spatial heterogeneity of LST requires the integration of remote sensing images, field surveys, and spatial statistical analyses to differentiate clustering regions (hot or cool spots) and identify influential factors (Barrell and Grant, 2013). Such clustering regions refer to areas with highly concentrated high or low LST over a period of time and have strong effects on the surrounding ecology and environment. The formation of these areas is closely related to ecological factors and human activities (Cañadas et al., 2014). The classification of clustering regions (hot-spot analysis) relies on the integration of geographical location and spatial relationships and requires spatial statistical analyses and models (Peeters et al., 2015). The degree of spatial clustering and spatial heterogeneity should be evaluated to reveal the spatial relationship between urban forest LST and multiple ecological factors. In contrast to classical statistical analyses, which typically omit spatial information, spatial statistical methods are suitable for analyzing the formation mechanisms of clustering regions by emphasizing the geographic variation in urban forest LST at temporal and spatial scales. Although this method has widely been used in other fields (e.g., geography, humanities, social sciences, and medicine), it has not been widely employed in environmental ecology (Ahmad et al., 2015).

The purpose of this study was to reveal how human activities and multiple ecological factors jointly influence urban forest LST through the analysis of clustering regions (hot and cool spots). Two main questions were addressed: 1) In addition to human activity, what are the main ecological factors influencing urban forest LST? 2) How do interactions between human activity and multiple ecological factors influence urban forest LST? We proposed a new quantitative method that integrates remote sensing, field surveys, and spatial statistical analyses, and chose the urban forest of Xiamen City, a representative Chinese city undergoing rapid urbanization, for a case study (Appendix D, Fig. 1). This work provides a scientific basis for the simulation and prediction of the spatial patterns of urban cold islands (Zhao et al., 2016a).

\section{Materials and methods}

\subsection{Overview}

The methodology consisted of the following three steps (Fig. 1). 1) A spatial database was created by integrating multi-source data, including forest management planning inventory (FMPI), digital elevation models (DEM), population, and remote sensing images. 2) LST spatial distribution data were calculated using thermal remote sensing images. Forest patch and LST data were integrated spatially. Hot and cool spots were identified on an appropriate threshold distance scale using spatial statistical analysis. 3) The results from clustering regions, multi-source spatial data, and the GeogDetector model were combined to reveal the driving mechanism of urban forest LST. For an overview of the study area, data sources, and other research methods, please see the overview in Appendix A.

\subsection{Multi-source spatial database development}

The multi-source data in 1996 and 2006 mainly included three types. The first type was FMPI data obtained by field observations, including graphical data and the corresponding attribute database. The attribute database provided data on stands, soil, topography, and other ecological factors, and the sampling accuracy was within $90 \%$. The second type was raster data of the population density distribution of Xiamen City, which characterized human activity. The third type was Landsat-5 TM images. LST data from the summer were derived from the thermal infrared (TIR) band (10.40-12.50 $\mu \mathrm{m}$ ) of two Landsat 5 TM images (Row 119/Path 43) acquired on July 20,1996 and August 17, 2006. The two images were acquired at approximately 10:00 a.m. local time under clear atmospheric conditions. Our study focused on the summer period (i.e., from July to September) because the forest canopy is fully developed during this time and the contrast between the LST of the forest and surrounding urban construction land is typically highest. Thus, summer is the optimal time to study the forest cooling effect. Moreover, from the perspective of urban forest planning, the focus of cooling is most relevant during summer. In contrast, during winter, the heat island effect on human health is relatively small and some trees are bare. It is ideal to select images from close dates to discern the LST variation during different periods and determine the contributions of various environmental factors. However, due to limited remote sensing image archives and the cloud-prone climate in southern China, only two qualified scenes (i.e., July 20, 1996 and August 17, 2006) under relatively clear and stable atmospheric conditions were selected (Appendix A, Data source).

With FMPI patches defined as the basic unit, the temporal and spatial distribution of population density was mapped using the kernel density tool and the zonal statistics function in the ArcGIS 10.0 spatial analysis module. Urban LST raster data were obtained by inversion using a single channel algorithm. The average values of population density and urban LST in each forest patch were calculated. The matched spatial data (temperature, population, and multiple ecological factors) were converted to the WGS 1984 UTM (50N) coordinate system. The readers are referred to Appendix A for details on the attribute database.

\subsection{Urban forest LST calculation}

\subsubsection{Data preprocessing}

First, the digital number (DN) values of the thermal band of the Landsat TM 5 were converted to radiance. Second, we used atmospheric correction methods to eliminate the influence of view angle and atmosphere on surface reflectance. Third, LST was estimated using the single-channel retrieval algorithm. Fourth, we used the 


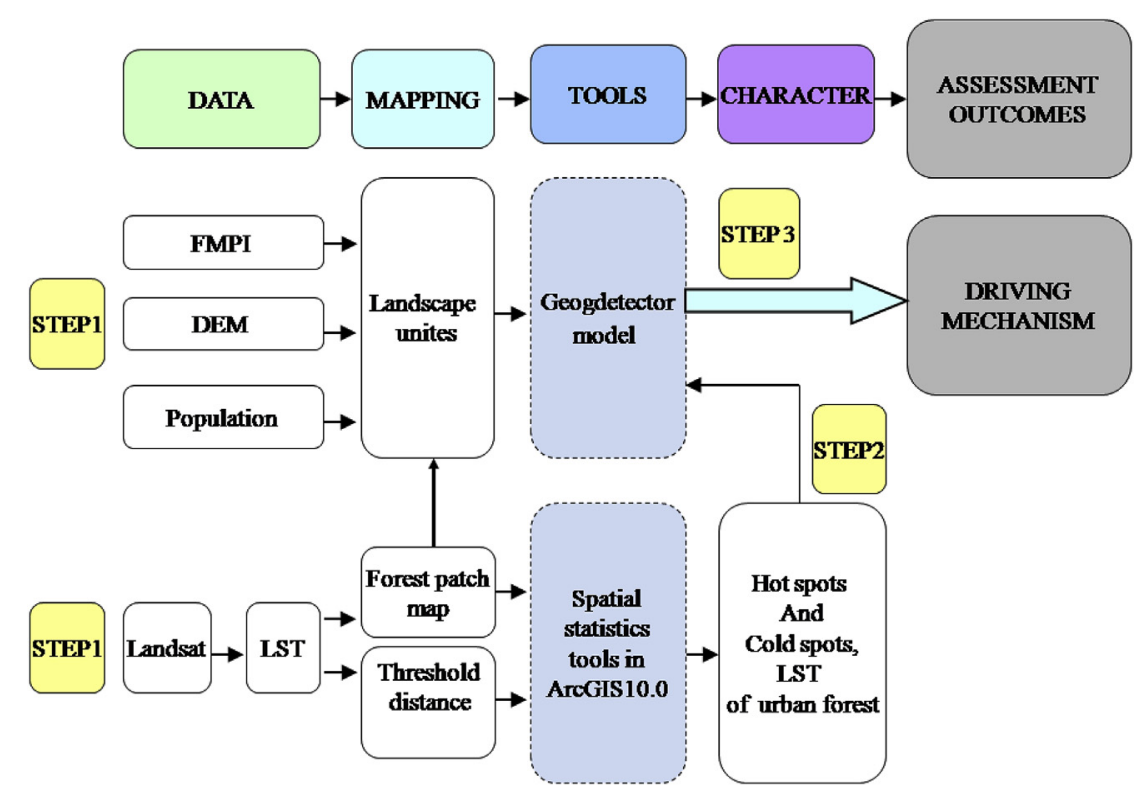

Fig. 1. Schematic of the proposed methodology. Data were processed using ArcGIS10.0 and the GeogDetector model.

cubic convolution interpolation method to resample the resolution from 120 to $30 \mathrm{~m}$ because the resolution of the Landsat TM 5 thermal infrared band was relatively coarse compared with the average area of the urban forest patches. Finally, the LST was normalized in order to evaluate the interactions among the multiple factors influencing urban forest temperature. Nondimensionalization can eliminate the influence of the viewing angle and different image acquisition times. A relative LST index $T_{R}$, proposed by Zhao et al. (2010), was used to normalize LST values as follows:

$\mathrm{T}_{\mathrm{R}}=\left(\mathrm{LST}-\mathrm{T}_{\mathrm{b}}\right) / \mathrm{T}_{\mathrm{b}}$

where LST is the surface temperature $(\mathrm{K})$ of each pixel and $\mathrm{T}_{\mathrm{b}}(\mathrm{K})$ is the background surface temperature defined as the average temperature of the study area images. See the supporting information for the detailed processes (Appendix A).

\subsubsection{Accuracy assessment}

Many studies have confirmed that the temperature difference between LST generated from remote sensing images and air temperature obtained from meteorological stations decreases with increasing NDVI (normalized difference vegetation index) (Weng et al., 2004; Yuan and Bauer, 2007). Thus, data measured at meteorological stations with known spatial locations were used for the urban forest LST test and a Pearson correlation analysis was conducted. We used the measured daily average air temperature data of 10 meteorological stations in Xiamen City to evaluate the accuracy of LST inversion (Appendix D, Fig 2). The Pearson correlation coefficient was used to evaluate the linear relationship between the estimated LST and observed air temperature (Li et al., 2011). The results showed that the estimated LST was correlated with observed air temperature (Pearson correlation coefficient: 0.848 ; $\mathrm{p}<0.01)$.

\subsection{Spatial statistical analysis}

\subsubsection{Determination of optimal threshold distance}

The optimal threshold distance was determined considering 1) the peak value of a variable (e.g., area and z-score) and 2) the number of significant patches (in hot or cool spots) in a neighboring area (mean and minimum numbers) based on the local Getis-Ord Gi $\times$ statistical results (Ord and Getis, 1995; Barrell and Grant, 2013). We calculated the compartment area and the neighboring number of significant patches by continuously increasing the threshold distance of each compartment (i.e., 500-m step increases in the 500-5000 $\mathrm{m}$ range) to find the maximum clustering distance (i.e., the optimal threshold). By increasing the threshold distance, the area of hot and cool spots first increased and then decreased (Table 1). When the threshold was in the range of $500-3500 \mathrm{~m}$, the area of hot and cool spots in 1996 and 2006 both increased and reached a maximum value at $3500 \mathrm{~m}$. Meanwhile, the mean neighboring values were always greater than 8 , which met the normal distribution assumption of autocorrelation. However, the minimum number did not exceed 8 until the distance reached the 2500-m threshold in 1996 (Ord and Getis, 1995). Therefore, we used $3500 \mathrm{~m}$, which met both the normal distribution assumption and the maximum area of hot and cold spots, as the optimal threshold (Table 1).

\subsubsection{Global Moran's I and local Getis-Ord Gi $\times$ statistical analysis}

The purpose of using global Moran's I and local Getis-Ord $\mathrm{Gi} \times$ statistics was to test the statistical significance at a given level and detect the spatial position of hot- and cool-spot clustering regions of urban forest LST on an optimal threshold-distance scale.

Global Moran's I is the most commonly used index for spatial autocorrelation analysis, and it is calculated with the following formula:

$I=\frac{N \Sigma_{i=1}^{n} \Sigma_{j=1}^{n}\left(X_{i}-\bar{X}\right)\left(X_{j}-\bar{X}\right)}{\left(\Sigma_{i=1}^{n} \Sigma_{j=1}^{n} W_{i j}\right) \Sigma_{i=1}^{n}\left(X_{i}-\bar{X}\right)^{2}}$

where $\mathrm{N}$ is the number of observed space units (polygonal region or point); $\bar{X}$ is the statistical mean; $X_{i}$ and $X_{j}$ are the observed value in the $\mathrm{i}$-th and $\mathrm{j}$-th region, respectively; and $W_{i j}$ is the weight of the $\mathrm{i}$ th region relative to the $\mathrm{j}$-th region.

Local Getis-Ord $\mathrm{Gi} \times$ is a local spatial statistical method that estimates the ratio of local values with different weights to the overall value based on distance measurement standards only, 
(a) 1996
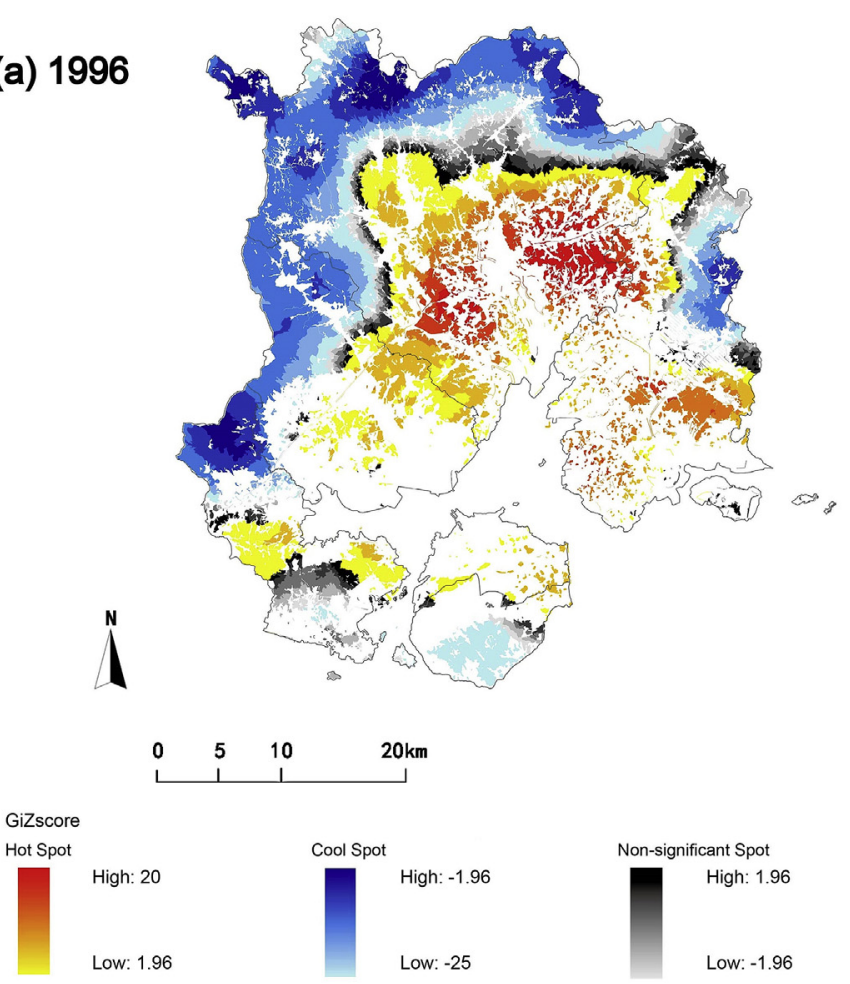

(b) 2006
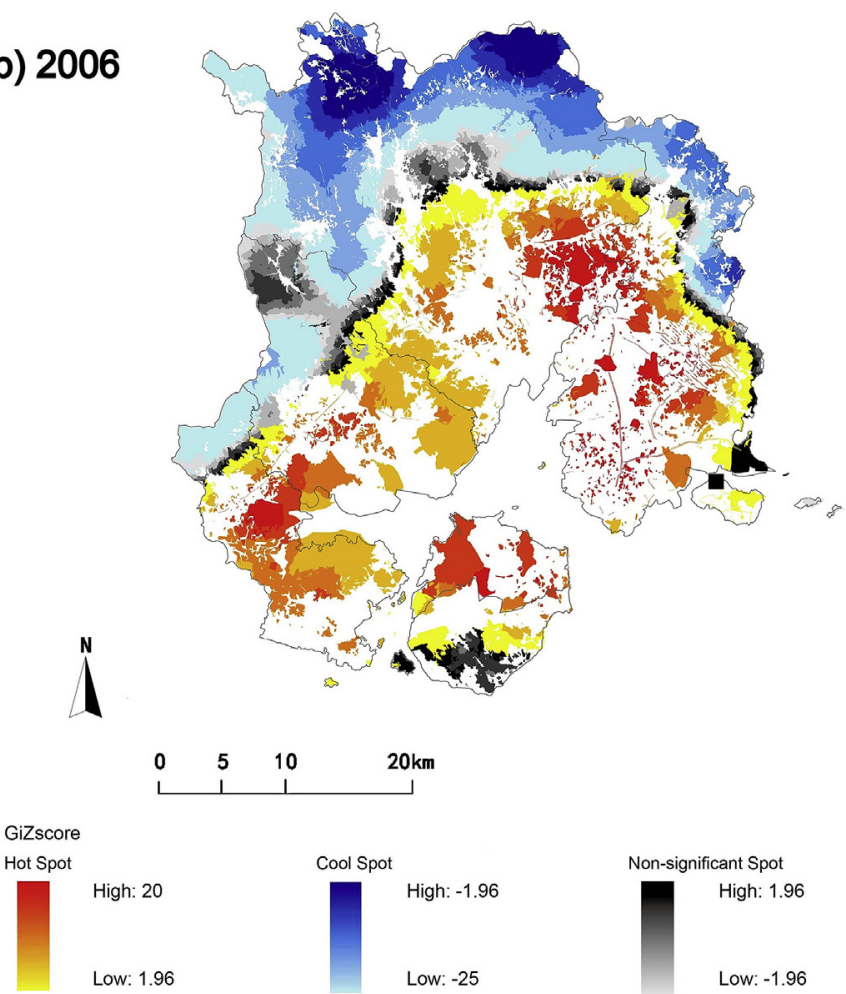

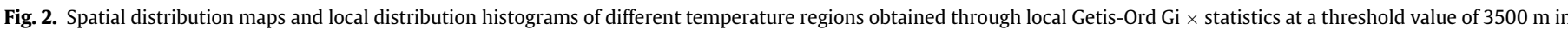

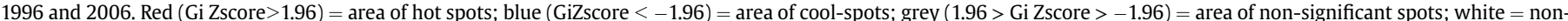
forest land. (For interpretation of the references to colour in this figure legend, the reader is referred to the web version of this article.)

Table 1

Results of local Getis-OrdG $\times$ analysis at ten thresholds identified as the total area, mean and minimum neighbors of hot and cool spots in 1996 and 2006

\begin{tabular}{|c|c|c|c|c|c|c|c|c|}
\hline \multirow[t]{2}{*}{ Thresholds (m) } & \multicolumn{2}{|c|}{ Areas of hot spots (ha) } & \multicolumn{2}{|c|}{ Areas of cool spots (ha) } & \multicolumn{2}{|l|}{ Mean neighbors(SD) } & \multicolumn{2}{|c|}{$\begin{array}{l}\text { Minimum(Maximum } \\
\text { neighbors) }\end{array}$} \\
\hline & 1996 & 2006 & 1996 & 2006 & 1996 & 2006 & 1996 & 2006 \\
\hline 500 & 8870.04 & $11,881.41$ & $11,881.41$ & $12,881.96$ & 41.978(27.584) & 47.193(36.351) & $3(365)$ & $3(235)$ \\
\hline 1000 & $17,599.47$ & $25,951.14$ & $28,186.95$ & $23,942.72$ & 149.661(80.072) & 204.524(99.217) & $3(519)$ & $7(523)$ \\
\hline 1500 & $20,524.81$ & $29,866.34$ & $32,819.41$ & $30,415.74$ & $254.553(129.622)$ & $356.025(157.762)$ & $3(600)$ & $20(774)$ \\
\hline 2000 & $22,769.57$ & $34,534.36$ & $34,604.52$ & $33,018.32$ & $382.614(182.799)$ & $547.685(224.356)$ & $5(677)$ & $34(1112)$ \\
\hline 2500 & $23,588.30$ & $36,598.21$ & $36,049.18$ & $35,282.95$ & $526.422(236.714)$ & 775.431(299.343) & $9(1477)$ & $54(1585)$ \\
\hline 3000 & $24,237.71$ & $37,189.53$ & $36,930.76$ & $37,006.52$ & 691.892(296.344) & $1035.21(381.458)$ & $29(1836)$ & $87(1963)$ \\
\hline 3500 & $25,440.14$ & $38,469.53$ & $37,889.81$ & $38,229.78$ & $874.477(351.539)$ & $1324.292(466.504)$ & $118(2097)$ & $124(2312)$ \\
\hline 4000 & $23,640.97$ & $37,763.26$ & $37,450.60$ & $36,539.46$ & $900.711(362.085)$ & $1721.579(606.407)$ & $154(2352)$ & $154(2636)$ \\
\hline 4500 & $23,533.87$ & $36,272.65$ & $36,990.06$ & $35,537.83$ & $990.783(398.295)$ & $1854.009(653.056)$ & $179(2925)$ & $183(2985)$ \\
\hline 5000 & $23,365.79$ & $36,178.55$ & $34,405.84$ & $34,905.91$ & $1060.138(426.177)$ & $2118.867(746.347)$ & $211(3028)$ & $225(3377)$ \\
\hline
\end{tabular}

without considering the condition of boundary adjacency. Its formula is as follows:

$G i^{*}(d)=\frac{\Sigma_{j=1}^{n} W_{i j}(d) X_{j}}{\Sigma_{j=1}^{n} X_{j}}$

where $d$ is the region size; $W_{i j}$ is the weight matrix of sample points and region $\mathrm{j}$; and $\mathrm{x}$ is an indicator quantifying importance.

\subsubsection{Definition of regions with various LST features}

By setting the optimal threshold distance as $3500 \mathrm{~m}$, a clustering graph was generated using the local Getis-Ord $\mathrm{Gi} \times$ index. According to the $\mathrm{z}$-score, regions with an absolute value greater than 1.96 were considered clustering regions (hot or cool spots). Areas with an absolute value less than 1.96 were considered nonsignificant (Mahboubi et al., 2015).
Three types of spatial patterns were identified and defined as follows:

(1) Hot spot: locations of statistically significant clustering of high LST values ( $z \geq 1.96$ refers to the upper limit of the 95\% confidence interval).

(2) Cool spots: locations of statistically significant clustering of low LST values $(z \leq-1.96$ refers to the lower limit of the $95 \%$ confidence interval).

(3) Non-significant spots: locations showing no significant spatial correlation $(1.96>\mathrm{z}>-1.96)$.

A high z-score and low p-value indicate spatial clustering of high values. A low, negative $\mathrm{z}$ value and a low $\mathrm{p}$-value indicate spatial clustering of low values. For significant positive z-scores, a higher z-score corresponds to a more sensitive degree of clustering 
of high values (Ivajnšič et al., 2014) (Appendix A).

\subsubsection{GeogDetector model description}

The GeogDetector model was used to detect various factors that may influence urban forest LST, the degree of influence of each factor, and the interactions between factors based on spatial analysis of variance (Wang and Hu, 2012). The GeogDetector model quantitatively expresses various types of interactions strengths among different ecological factors. The model follows a spatial heterogeneity hypothesis-based approach and is suitable for ecological study areas with strong spatial heterogeneity. The GeogDetector model includes factor, ecological, and interaction detectors. The factor detector is used to explore the impact of different factors on the research target; the ecological detector is used to explore the impacts of different levels of significance on those factors; and the interaction detector is used to explore the strengths of combinations of different impact factors on the research target. The GeogDetector model is freely available from www.sssampling. org/geogdetector.

Assuming that human activities and certain ecological factors jointly influence urban forest LST, the temporal and spatial distribution pattern of LST could then be determined. We first loaded map layers of all human and ecological factors and urban forest LST values into ArcGIS. We extracted the average values of the ELE (elevation), SDe (slope degree), and PD (population density) factors based on forest patches. The data was classified according to the classification criteria of the FMPI instruction manual (e.g., SDe); the factors not classified in the manual were classified using the Natural Breaks (Jenks) method (e.g., PA or patch area). The average LST of each forest patch was set as the dependent variable in the statistical analysis. Multiple ecological and human activity factors deemed as independent variables were introduced into GeogDetector model. All the different impact factor values were input into the GeogDetector model for simulations. For additional details, please see Appendix A, Spatial statistical analysis.

\section{Results}

\subsection{Urban forest LST and stand structure}

Landsat 5 images from 1996 to 2006 were combined with the corresponding FMPI data $(n=14,901$ in 1996 and $n=14,944$ in 2006). A multi-source dataset, in which the LST of each forest patch corresponds to various ecological factors, was generated on the same urban scale. In 1996, 2006, the relative average urban forest LST was -0.014 and-0.042, respectively (Fig. 2).

The stand structure varied greatly from 1996 to 2006. Specifically, the clustering degree increased by 0.118 (Moran's index was 0.155 in 1996 and 0.273 in 2006) (Table 2), the total area of forest patches increased by $12.227 \%(68,559.733$ ha in 1996; $89,279.067$ ha in 2006; total area of $165,036.300$ ha) (Appendix C, Table 1), the average forest age increased by $1.877 \mathrm{yr}$ $(16.066 \pm 11.758 \mathrm{yr}$ in $1996 ; 17.943 \pm 10.271 \mathrm{yr}$ in 2006$)$, and the average canopy density increased by $0.046(0.229 \pm 0.276$ in 1996 ; $0.275 \pm 0.297$ in 2006). The dominant tree species in 1996 were

Table 2

Global spatial autocorrelation statistics (Moran's I) of urban forest LST in 1996 and 2006.

\begin{tabular}{lll}
\hline Year & 1996 & 2006 \\
\hline Moran's Index & $0.155^{* *}$ & $0.273^{* *}$ \\
z-score & 244.504 & 432.03 \\
Pattern & Clustered & Clustered \\
\hline
\end{tabular}

Note: $\mathrm{P}<0.001$ in all regions in 1996 and 2006; ${ }^{* *}$ represent significant values.
Dimocarpus longan, Pinus massoniana, Acacia confuse, Pinus elliottii, Cunninghamia lanceolata, Camellia sinensis, Casuarina equisetifolia, and Schima superba. The same species were dominant in 2006, in addition to Eucalyptus spp. The planting area of dominant tree species changed. For example, the area of Acacia confuse increased by $1.764 \%$ (5.148\% in 1996; $6.912 \%$ in 2006), the area of P. massoniana decreased by $9.821 \%$ (34.475\% in $1996 ; 24.654 \%$ in $2006)$, and the area of $D$. longan decreased by $8.165 \%(28.096 \%$ in 1996; 19.931\% in 2006) (Fig. 3, Appendix C, Table 2).

\subsection{Population density}

The population density in Xiamen City increased from $565.0 \pm 12.6$ persons/ha in 1996 to $755.5 \pm 11.9$ persons/ha in 2006 ( $t$-test, $\mathrm{p}<0.01$ ). Regions with different degrees of urbanization all showed an increasing trend. However, the degree of population aggregation tended to decrease, as seen in Fig. 4 (Moran's index $=0.348$, z-score $=650.417$ in 1996; Moran's index $=0.331, z$ score $=532.505$ in 2006). The results based on classification through local Getis-Ord Gi $\times$ statistics show that hot and cool spots of population and LST showed similar temporal and spatial distribution patterns. For example, $92.831 \%$ of population hot spots and $64.115 \%$ of population cool spots overlapped with corresponding areas of urban forest LST in 2006.

\subsection{Influence of human activities and multiple ecological factors on urban forest LST}

After classification through local Getis-Ord $\mathrm{Gi} \times$ statistics, the GeogDetector model was used to compare hot and cool spots of urban forest LST. Stand and topography groups were dominant ecological factors that influenced forest LST in 1996 and 2006. In particular, dominant tree species (stand factor) and elevation (topography factor) had greater impacts in different clustering regions and time periods compared with population density (Fig. 5, Table 3). In addition, during different time periods and in different clustering regions, linear or nonlinear interactions were observed for all relationships between population density and the 11 selected factors: (population density $\cap$ ecological factor) $>$ (population density + ecological factor) or (population density $\cap$ ecological factor) $>$ (population density, ecological factor) (Table 4, Appendix $C$, Table 3). There were strong interaction effects between the dominant factor and other ecological factors. In particular, strong interactions between two ecological factors were generally observed in the elevation (ELE) and dominant species (DS) (Fig. 6).

Ecological factors had varying impacts on forest LST in different clustering regions and during different time periods. For example, in 2006, the main influential factors in hot spots were elevation $(q=0.139)$, slope $(q=0.129)$, dominant tree species $(q=0.099)$, and forest patch area $(q=0.085)$; the main influential factors in cool spots were dominant tree species $(\mathrm{q}=0.07)$, elevation $(\mathrm{q}=0.069)$, population density $(\mathrm{q}=0.069)$, and canopy density $(q=0.036)$ (Table 3). Moreover, significantly interacting ecological factors varied among hot and cool spots. For example, population density significantly interacted with forest patch area, forest age, and elevation in hot spots. In cool spots, population density significantly interacted with canopy density, soil quality, soil thickness, humus depth, slope, slope direction, and slope position (Table 4). In addition, the factors with strong interaction effects changed from hot to cold spot areas during different years. Specifically, in 1996, elevation and the dominant species were dominant interaction effect factors in hot and cold spots of urban forests, respectively. In contrast, dominant species and elevation became the dominant interaction factors in hot and cool spots, respectively, in 2006. (Fig. 6, Appendix C, Table 4). 

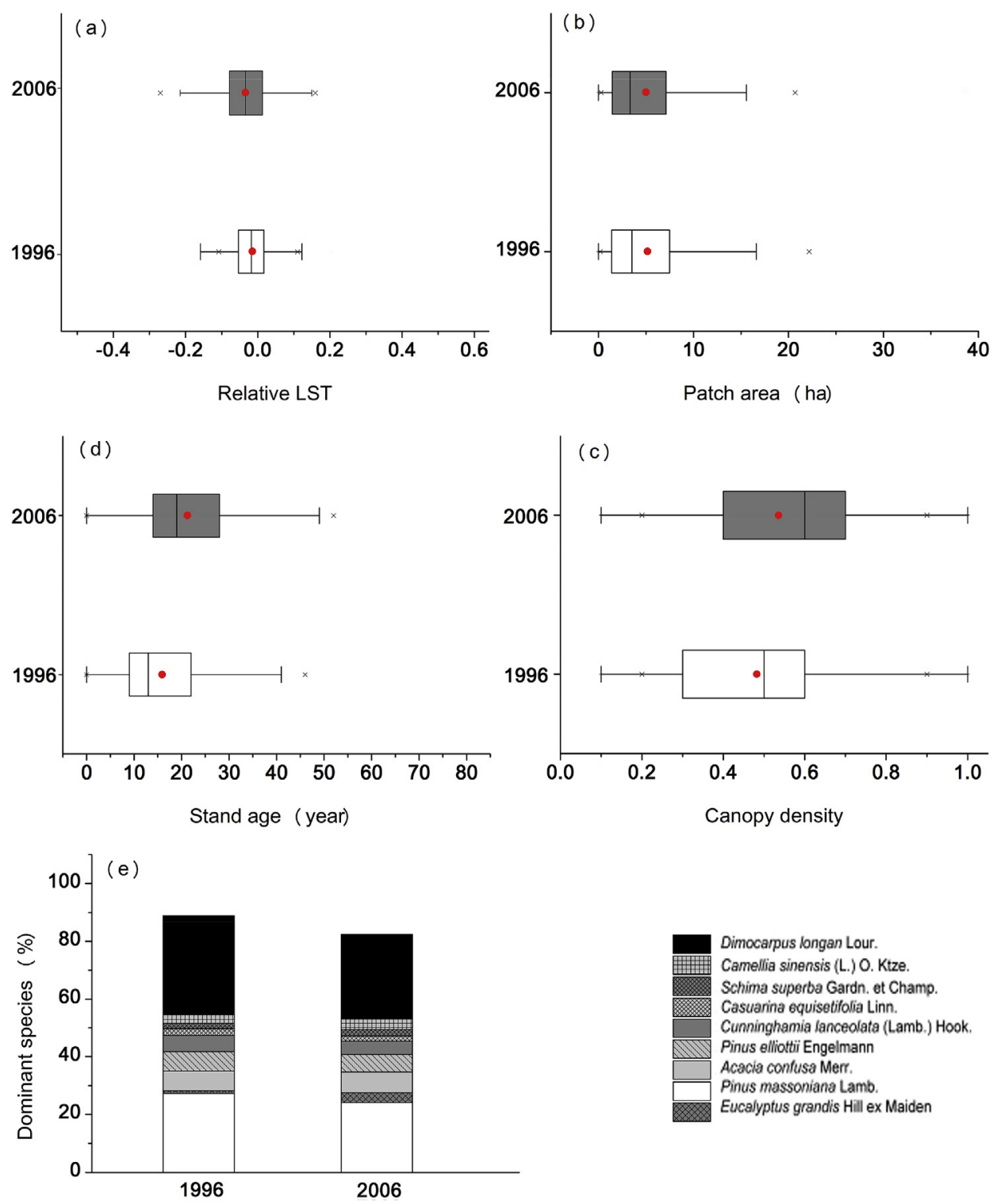

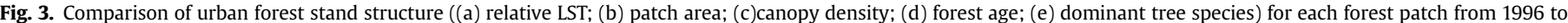

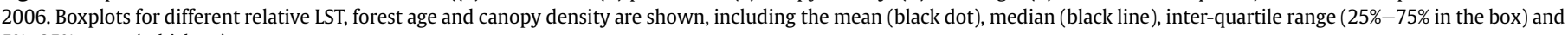
$5 \%-95 \%$ range (whiskers).

\section{Discussion}

\subsection{Interaction effects on urban forest LST}

In this study, remote sensing imagery, FMPI, spatial statistical analysis, and GIS technology were integrated to reveal the complicated interactions (direct and indirect) between various ecological factors and their effects on urban forest LST. Two key properties of these interactions were addressed: strength and direction. We identified the strength of interactions between two ecological factors and their impact on spatial heterogeneity of urban forest LST. Furthermore, the interaction strengths showed that elevation and dominant species had a greater influence on urban forest LST in comparison to other factors (e.g., patch area). In addition, elevation and dominant species displayed strong interactions with other ecological factors and linearly or non-linearly enhanced the urban forest cooling effect.

Our results confirm that elevation had a direct and dominant impact on urban forest LST and displayed strong interactions with multiple ecological factors (e.g., dominant species, population density). This indicates that elevation, which controls urban vegetation growth by changing light, moisture, and soil nutrients, and thus influences urban forest LST, is the major cause of spatial heterogeneity of urban LST. Recently, many studies have revealed interactions between elevation and vegetation cover, soil depth, and vegetation type using multiple linear regression models and plotscale transplanting experiments in natural ecosystems (Grassein et al. 2014). Tayyebi and Jenerette (2016) proposed that further research on the interactions of urban forest LST and elevation at the city scale can alleviate regional and global warming and provide a scientific basis for addressing climate change adaptation policies. However, due to the lack of a database of multi-source data, routine experimentation and empirical equations cannot adequately quantify the complex non-linear interactions between elevation and multiple factors on an urban scale. This leads to difficulty in elucidating the quantitative interactions between elevation and 
(a) 1996

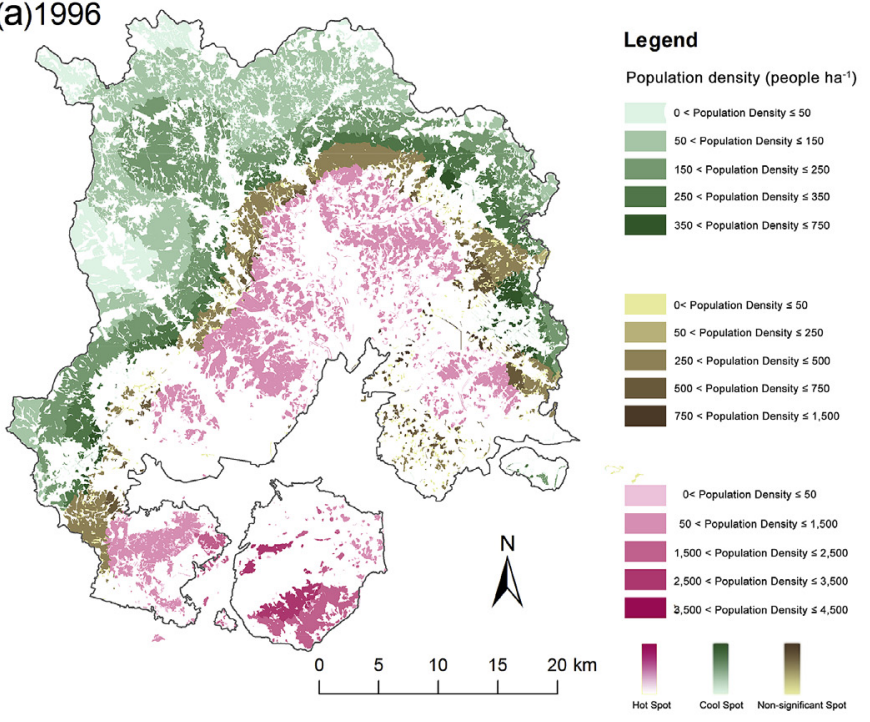

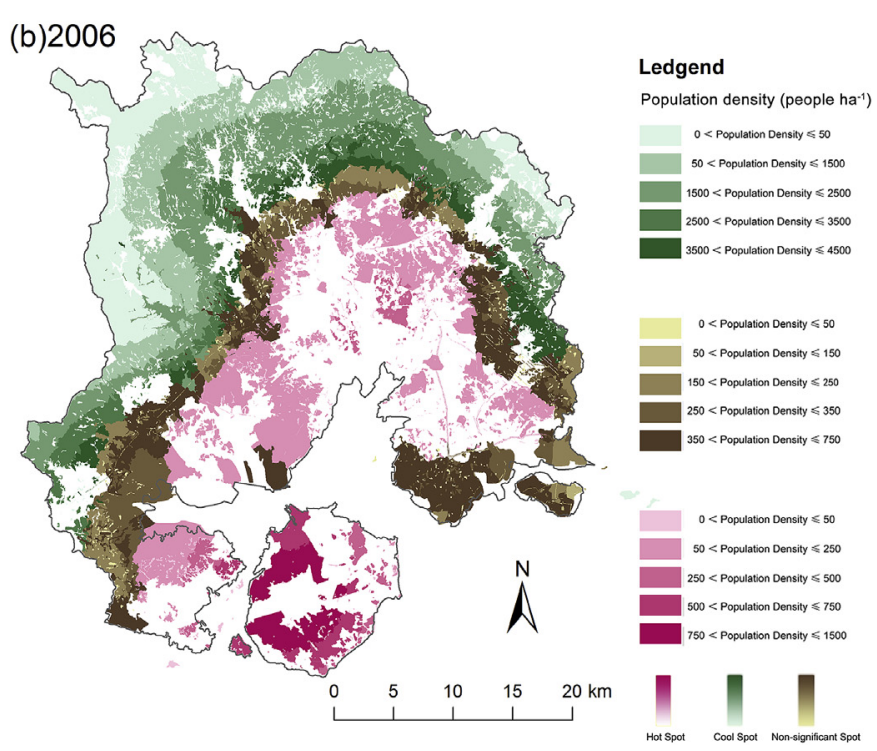

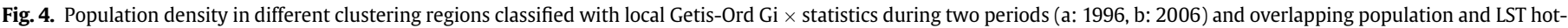

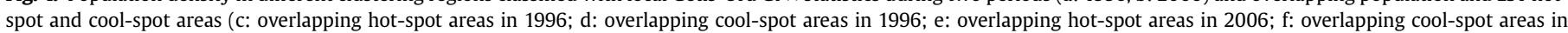
2006).

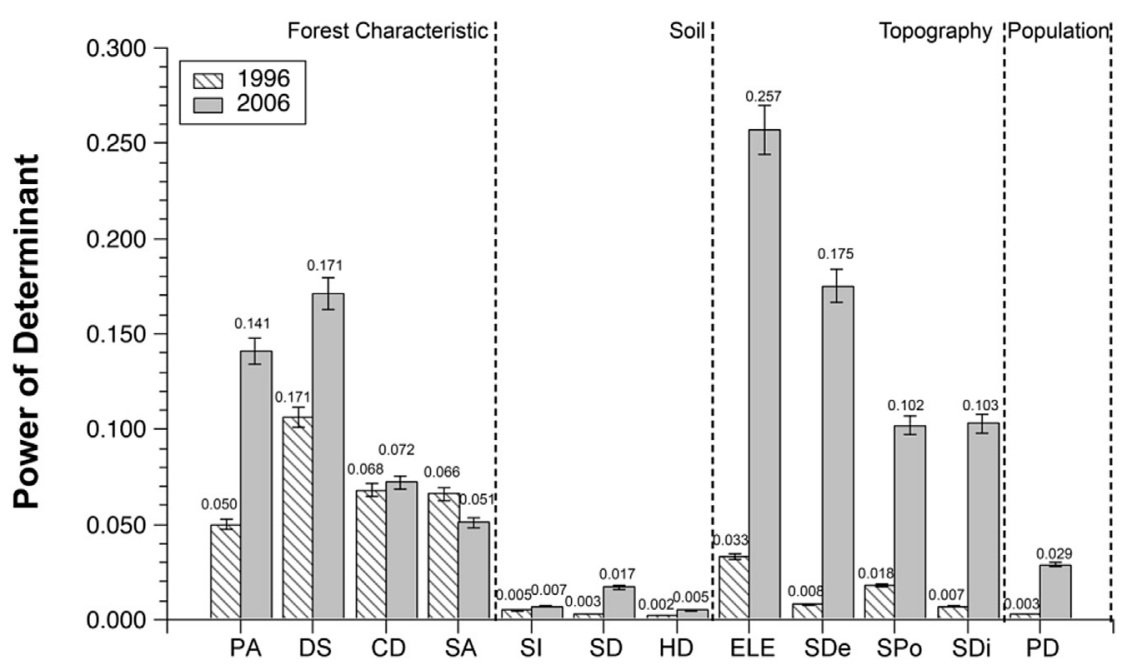

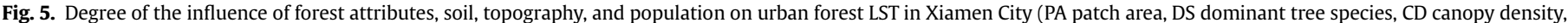
SA stand age, SI site index, SD soil depth, HD humus depth, ELE elevation, SDe slope degree, SPo slope position, SDi slope direction, PD population).

Table 3

Degree of the influence of forest attributes, soil, topography, and population on urban forest LST in the entire city and three temperature regions.

\begin{tabular}{|c|c|c|c|c|c|c|c|c|c|}
\hline & & \multicolumn{2}{|l|}{ ALL } & \multicolumn{2}{|l|}{$\mathrm{HS}$} & \multicolumn{2}{|l|}{$\mathrm{CS}$} & \multicolumn{2}{|l|}{ NS } \\
\hline & & 1996 & 2006 & 1996 & 2006 & 1996 & 2006 & 1996 & 2006 \\
\hline \multirow[t]{4}{*}{ Forest attributes } & PA & 0.050 & 0.141 & 0.020 & 0.085 & 0.004 & 0.015 & 0.001 & 0.029 \\
\hline & DS & 0.106 & 0.171 & 0.042 & 0.099 & 0.063 & 0.070 & 0.067 & 0.125 \\
\hline & $\mathrm{CD}$ & 0.068 & 0.072 & 0.033 & 0.064 & 0.030 & 0.036 & 0.066 & 0.095 \\
\hline & SA & 0.066 & 0.051 & 0.032 & 0.061 & 0.026 & 0.017 & 0.047 & 0.082 \\
\hline \multirow[t]{3}{*}{ Soil } & SI & 0.005 & 0.007 & 0.001 & 0.009 & 0.004 & 0.004 & 0.006 & 0.011 \\
\hline & SD & 0.003 & 0.017 & 0.003 & 0.035 & 0.002 & 0.008 & 0.002 & 0.011 \\
\hline & HD & 0.002 & 0.005 & 0.002 & 0.009 & 0.004 & 0.000 & 0.002 & 0.002 \\
\hline \multirow[t]{4}{*}{ Topography } & ELE & 0.033 & 0.257 & 0.045 & 0.139 & 0.033 & 0.069 & 0.006 & 0.079 \\
\hline & SDe & 0.008 & 0.175 & 0.021 & 0.129 & 0.012 & 0.025 & 0.005 & 0.076 \\
\hline & SPo & 0.018 & 0.102 & 0.011 & 0.061 & 0.067 & 0.027 & 0.007 & 0.072 \\
\hline & SDi & 0.007 & 0.103 & 0.009 & 0.075 & 0.012 & 0.023 & 0.007 & 0.060 \\
\hline Population & PD & 0.003 & 0.029 & 0.005 & 0.007 & 0.005 & 0.069 & 0.003 & 0.007 \\
\hline
\end{tabular}

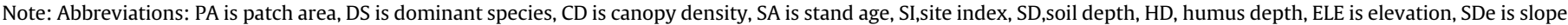
degree, SPo is slope position, SDi is slope direction, SI is site index, and PD is population density. 
Table 4

Influence of interactions between population density and other ecological factors in hot spots, cool spots, and non-significant spots in 1996 and 2006.

\begin{tabular}{|c|c|c|c|c|c|c|c|c|}
\hline & \multicolumn{4}{|l|}{1996} & \multicolumn{4}{|l|}{2006} \\
\hline & All & Hot spot & Cool spot & Non-significant spot & All & Hot spot & Cool spot & Non-significant spot \\
\hline PA & $0.061 * \nearrow \nearrow$ & $0.036 \nearrow \nearrow$ & $0.021 \nearrow \nearrow$ & $0.010 \nearrow \nearrow$ & $0.201^{*} \nearrow \nearrow$ & $0.106^{*} \nearrow \nearrow$ & $0.083 \uparrow$ & $0.066 \nearrow \nearrow$ \\
\hline DS & $0.129 \nearrow \nearrow$ & $0.063 \nearrow \nearrow$ & $0.087 \nearrow \nearrow$ & $0.105 \nearrow \nearrow$ & $0.244 \nearrow \nearrow$ & $0.143 \nearrow \nearrow$ & $0.141 \nearrow \nearrow$ & $0.169 \nearrow \nearrow$ \\
\hline $\mathrm{CD}$ & $0.077 \nearrow$ & $0.048 \nearrow \nearrow$ & $0.038 \nearrow \nearrow$ & $0.105 \nearrow \nearrow$ & $0.118 \nearrow \nearrow$ & 0.082 メ & $0.105^{*}$ オ & $0.117 \nearrow \nearrow$ \\
\hline SA & 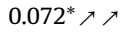 & $0.048 \nearrow \nearrow$ & $0.034 \nearrow \nearrow$ & $0.059 \nearrow \nearrow$ & $0.115^{*} \nearrow \nearrow$ & $0.083^{*} \nearrow \nearrow$ & $0.086 \nearrow \nearrow$ & $0.109 * \nearrow \nearrow$ \\
\hline SI & $0.007 \uparrow$ & $0.006 \nearrow \nearrow$ & $0.007 \uparrow$ & $0.016 \nearrow \nearrow$ & $0.040 * \nearrow \nearrow$ & $0.022 \nearrow \nearrow$ & $0.074^{*} \nearrow \nearrow$ & $0.023 \nearrow \nearrow$ \\
\hline SD & $0.006 \nearrow \nearrow$ & $0.008 \nearrow \nearrow$ & $0.006 \nearrow \nearrow$ & $0.013 \nearrow \nearrow$ & $0.056 \nearrow \nearrow$ & $0.046 \nearrow \nearrow$ & $0.081^{*} \nearrow \nearrow$ & $0.025 \nearrow \nearrow$ \\
\hline HD & $0.004 \nearrow \nearrow$ & $0.006 \uparrow$ & $0.005 \nearrow \nearrow$ & $0.026 \nearrow \nearrow$ & $0.039 \nearrow \nearrow$ & $0.016 \nearrow \nearrow$ & $0.070 * \nearrow \nearrow$ & $0.010 \nearrow \nearrow$ \\
\hline ELE & $0.0428 \nearrow \nearrow$ & $0.009 \nearrow \nearrow$ & $0.016 \nearrow \nearrow$ & $0.015 \nearrow \nearrow$ & $0.294^{*} \nearrow \nearrow$ & $0.155^{*} \nearrow \nearrow$ & $0.137 \uparrow$ & $0.103 * \nearrow$ \\
\hline SDe & $0.012 \nearrow \nearrow$ & $0.010 \nearrow \nearrow$ & $0.014 \nearrow \nearrow$ & $0.012 \nearrow \nearrow$ & $0.218 \nearrow \nearrow$ & 0.166 オ & $0.095 *$ オ & $0.113 \nearrow \nearrow$ \\
\hline SPo & $0.025 \nearrow \nearrow$ & $0.008 \nearrow \nearrow$ & $0.018 \nearrow \nearrow$ & $0.020 \nearrow \nearrow$ & $0.139 \nearrow \nearrow$ & $0.090 \nearrow \nearrow$ & 0.096 × オ & $0.101 \nearrow \nearrow$ \\
\hline SDi & $0.013 \nearrow \nearrow$ & $0.010 \nearrow \nearrow$ & $0.013 \nearrow \nearrow$ & $0.016 \nearrow \nearrow$ & $0.141 \nearrow \nearrow$ & $0.092 \nearrow \nearrow$ & $0.092 * \uparrow$ & $0.085 \nearrow \nearrow$ \\
\hline
\end{tabular}

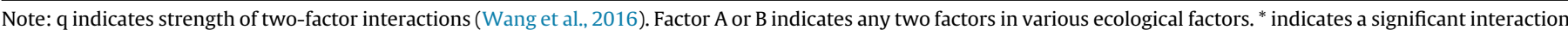

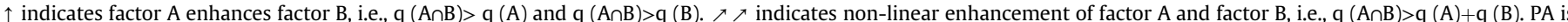

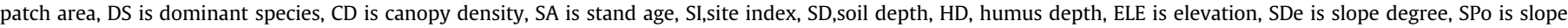
position, SDi is slope direction, SI is site index, and PD is population density.

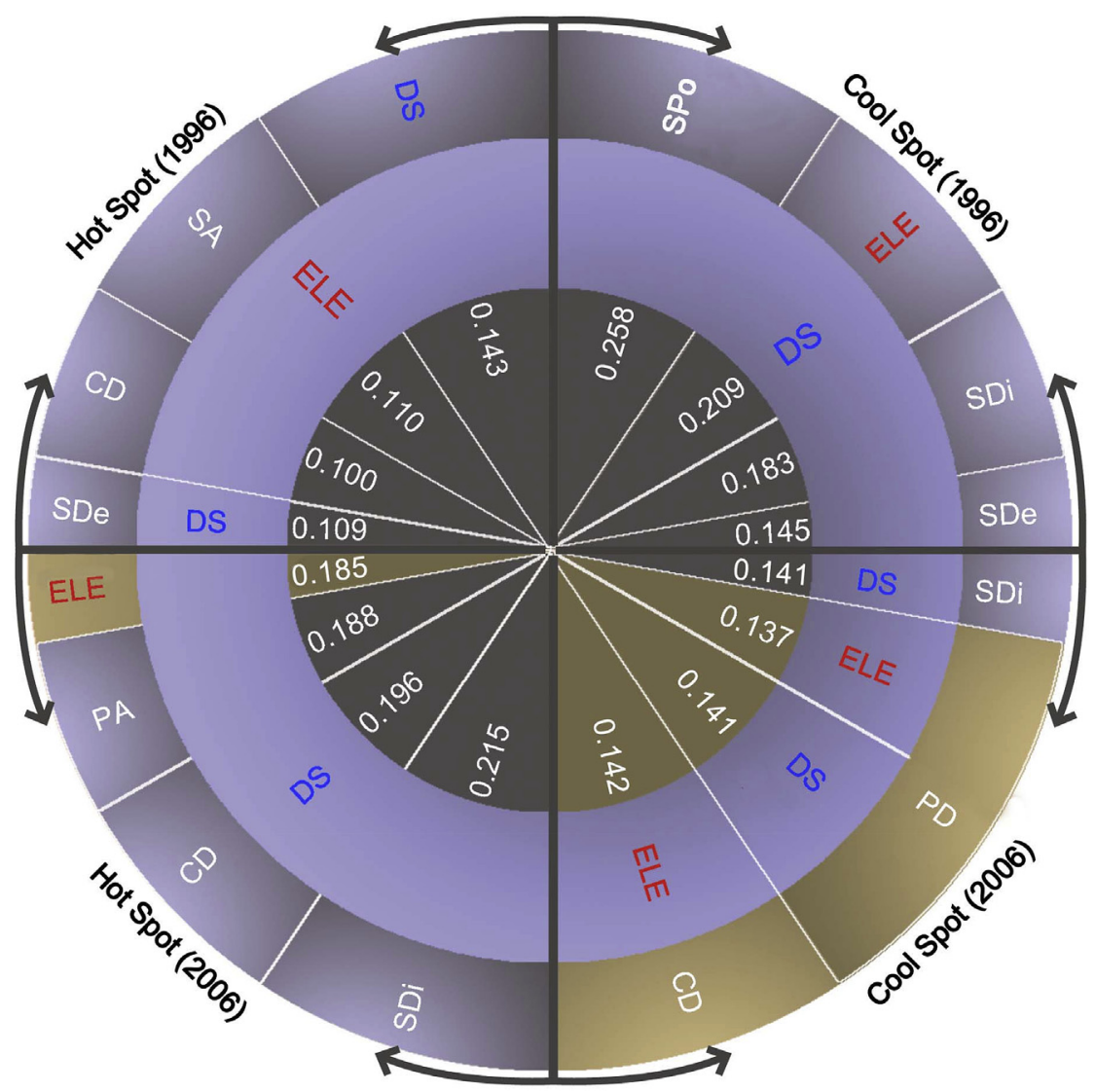

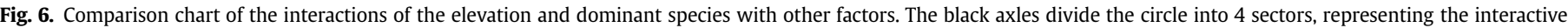

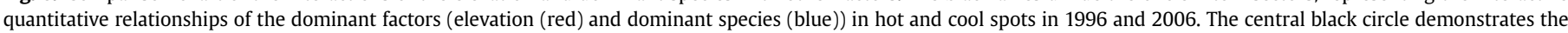

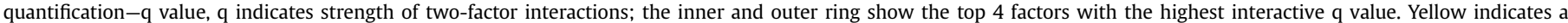

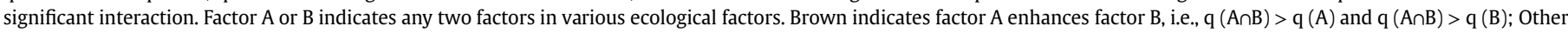

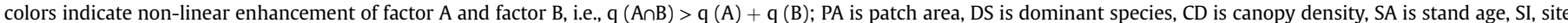

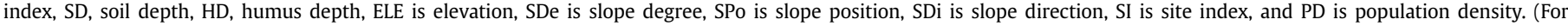
interpretation of the references to colour in this figure legend, the reader is referred to the web version of this article.)

urban forest structure (e.g., dominant species). Therefore, under scarce urban land resources, urban forest conservation and landscape planning should take into account elevation factors to enhance the urban forest cold island effect. Our results also show that interactions among dominant tree species and topographical factors (e.g., ELE, SDe, SPo) linearly or non-linearly enhanced the urban forest cooling effect. The results based on the GeogDetector model support the study by Long et al. (2015), which used an elevation gradient experiment in arctic tundra of Sweden to show that dominant tree species had more significant direct and interaction effects on temperature variation than other factors. This study indicated that the dominant tree species depends on a variety 
of factors including shape, breadth, crown length, clear length, tree morphology, density, leaf size, and leaf opacity, as well as interactions with topographical factors (e.g., ELE, SDe, SPo) and their effects on urban forest LST. Compared with elevation, few quantitative studies have focused on the interactions between species composition and multiple ecological factors on urban forest LST. Moreover, previous studies on the biological characteristics of the cooling effect due to different tree species have mainly been carried out on smaller scales such as in parks and sample plots (Lin et al., 2015). Our interaction study compares patches with different dominant trees species on an urban scale. For example, patches with $D$. longan as the dominant tree species mainly exist in hot spots, whereas those dominated by $S$. superba are mainly distributed in cool spots. This may be related to the above-mentioned biological factors. S. superba has thicker leaves and a higher and larger crown compared with $D$. longan trees of the same age. Norton et al. (2015) also demonstrated that dominant tree species are the main determinants of the cold island effect on a regional scale, and this is feasible in cities with limited land.

Although the interactions of elevation and dominant species with other ecological factors significantly affected urban forest LST in different clustering areas (hot and cold spots) and years, the strongest interaction factors varied. Specifically, in 1996, elevation and dominant species were the dominant factors showing interaction effects in hot and cold spots of urban forests, respectively. In contrast, dominant species and elevation became major interaction factors in hot and cool spots, respectively, in 2006. This change may be related to human willingness to reform urban forests, which varied among different spatial clustering regions. Human impacts in higher elevation regions varied among different years according to transportation accessibility. These changes resulted in variation in the interactions between elevation and other factors in the hot and cold spots. Moreover, large-scale afforestation changed the dominant species composition. Significantly different urban forest LST in hot and cold spots among different years may have been caused by interactions between tree species and other factors. The results of the study by Connors et al. (2013) confirm our point; they concluded that human activities caused positive and negative double impacts on urban forest LST, and LST was affected to various degrees by elevation and other topographical factors because of changes in urban forest structure among different locations and years. Interactions between these factors were complex, non-linear, and diverse. In our study, interactions between human activities and multiple ecological factors in hot and cool spots linearly or nonlinearly increased urban forest LST. Previous studies on urban LST mechanisms have quantified the relationships between human activities, mainly including landscape composition (e.g., vegetation coverage, impermeability, water proportion), spatial configuration (e.g., shape index, Shannon diversity index), and LST, whereas they only slightly consider human activities and the aforementioned factors. Nowadays, more and more urban forests are located in high population density regions. Human activities and other ecological factors modify urban forest spatial structure and composition and have inevitable large impacts on urban forest LST. The intervention is perennial and continuous (Josefsson et al. 2009). Kuang et al. (2014) proposed that using ecological process models for precise predictions of urban forest LST spatial distributions and variation trends under different human activity intensities can provide a scientific basis for urban forest landscape planning considering long-term cold island effects. Our research demonstrates that indirect effects generated by human activities elevate urban forest LST in highly heterogeneous urban forest ecosystems. Furthermore, the interaction between two factors can have a greater influence than the direct effect of a single ecological factor, and the interaction effect may even be greater than the combination of two factors' direct actions. As urban ecological processes advance, interactions will continue to grow and impede the cold island effect of urban forests. Therefore, the relationship between population distribution variations and urban forest construction management should be considered, in addition to interactions of elevation and dominant tree species with other factors.

In addition, although the influence of stand area on urban forest LST was relatively lower in 1996, we cannot simply infer that stand area is not important to urban forests' cooling effect. The influence of patch area on urban forest LST is closely related to the degree of forest fragmentation at a specific time and site and the connectivity between patches. More importantly, the indirect effect between patch area and multiple ecological factors has a stronger influence on urban forest LST than direct effects. In fact, when a strong interaction exists between ecological factors, statistical significance does not have true ecological significance when only considering the impact of dominant factors (Levy et al., 2014).

In recent years, numerous studies have emphasized that in order to clarify the driving mechanism of urban cool islands, the direction of interactions between ecological factors must be determined. The results of such studies are important for sustainable urban forest landscape planning (Kuang et al., 2014; Long et al., 2015). In our study, Pearson's correlation analysis was used to determine directions, where the direction of the Pearson's correlation coefficient represents the direction of a single-factor direct effect for urban forest LST (Appendix Table 5). For example, canopy density was significantly negatively related to the relative LST index in 1996 and $2006(P<0.01)$, indicating that the relative LST index moved in the negative direction with a changing canopy density. However, the relationship between two ecological factors is nonlinear in urban ecosystems; thus, the direction of a single factor could not be used to infer the direction of a two-factor interaction. In addition, the statistical software (e.g., SPSS) was inadequate for analyzing the types of variables investigated in our study (e.g., dominant tree species composition; the order of numbers assigned had no real meaning). As seen from current literature, the issue of interaction direction has become a difficult and hot topic in ecology. To our knowledge, the development of models can hardly solve the issue. Similarly, the GeogDetector model does not have a function to deal with the direction of two-factor interactions. However, GeogDetector has obvious advantages over traditional software that are only able to use a certain type of variable, which constrains data input. In contrast, the GeogDetector model processes both type and numeric variables, in parallel to solving all types of quantitative calculation problems. In addition, the GeogDetector model quantitatively expresses various types of interaction strengths among different variables. Most importantly, the GeogDetector model is a spatial heterogeneity hypothesis-based approach, as ecological study areas have strong spatial heterogeneity. Some studies have shown that the solution to interaction direction can be achieved by combining statistical analyses and spatial heterogeneity of environmental conditions (Heffernan et al., 2014; Tayyebi and Jenerette, 2016). For instance, Grassein et al. (2014) integrated R software and spatial heterogeneity of environmental conditions, to demonstrate that biotic interactions can affect plant performance but their direction depends on local environmental conditions. In our results, the spatial interactions between any two ecological factors, as well as their linearly enhanced or nonlinearly enhanced urban forest LST, were revealed. Therefore, the interaction direction may be determined based on the spatial heterogeneity results in the future. The GeogDetector model may be applied to study the effects of multiple ecological factors when analyzing interaction directions in spatially heterogeneous systems.

In summary, because of limited land resources and frequent 
human activities in urban areas, urban forest planning should used to create urban cool islands. A multi-source spatial database should be constructed and be used for urban forest planning. In addition, spatial statistical analyses should be used to differentiate clustering regions. In particular, the strengths and directions of interactions between any two ecological factors in urban forest patches should be quantitatively analyzed. Policies that protect landscape based on urbanization stage, clustering regions, and driving mechanisms of urban forest LST should take into account direct and indirect effects of various ecological factors. For instance, according to the results of interaction strengths, urban forest planning should consider the change due to elevation in hot spot areas in 1996. In contrast, changes in species composition should focus on urban forest planning in hot spot areas in 2006.

\subsection{Recommendations and future studies}

We put forward the following three suggestions according to our research results. First, to solve regional-scale urban environmental problems, we need to combine the respective advantages of multi-source data and spatial statistical analysis, and explore the interaction effects of human activities, LST, and multiple ecological factors, rather than only focus on qualitative descriptions of urban forest spatial structure. Moreover, we need to integrate information on macro-spatial patterns and micro-urban patches and build a multi-source database with a unified spatial-temporal scale. Second, more emphasis should be given to studies on identifying influential factors in clustering regions of urban forests. The degree and location of changes in these areas should be determined to reveal variation due to urbanization. Finally, urban forest planning should pay close attention to the strength and direction of interactions between human activity, elevation, and dominant tree species on urban forest LST spatial heterogeneity and divide different functional clustering regions considering each ecological factors' direct and indirect (interacted) effects.

Our study quantitatively compares the interaction effects at two single time points. We propose the following future studies based on our results. 1) Future studies should take into account the temporal and spatial heterogeneity of LST. Quantitative studies on heterogeneity should be carried out on different temporal and spatial scales, in different cities and seasons, and with remote sensing images of various resolutions; 2) Accurate LST inversion methods should be used instead of the nondimensional relative temperature to overcome uncertainties due to field view, weather conditions, etc. The integration of time series Landsat images on the same urban scale remains to be further studied; 3) The limited ecological factors selected in this study cannot fully express the driving mechanism of LST. Some of the selected ecological factors (e.g., species composition, and elevation) displayed dominant influences on urban forest LST, but the direct effects and degree of interaction of these factors in different regional environments and urbanization stages may vary; 4) The development of the GeogDetector model should focus determining the direction of interactions between ecological factors, and strength and direction of interactions should be applied to urban forest planning; and 5) The environmental impacts of combined pollution on urban forest health should be considered. Previous studies have shown that pollutants such as cement ash, fly ash, limestone dust, and other industrial particulate matter (dust pollution) settle onto plant leaf surfaces in different urban surface temperature (thermal pollution) environments. These pollutant have various impacts on plant growth indices, including leaf stomatal conductance, chlorophyll content, electron transfer rate, leaf temperature, photosynthesis rate, respiration rate, and transpiration rate (Naidoo and Chirkoot, 2004; Van Heerden et al., 2007).

\section{Conclusions}

Our results show that during rapid urbanization, the degree of spatial aggregation of urban forest LST increased. Hot spots were mainly distributed in the urban core and suburbs, and cool spots were mainly located in suburbs and outer suburban districts. Compared with human activities, dominant tree species and elevation were the dominant factors influencing urban forest LST. The interactions between human activity and specific ecological factors linearly or nonlinearly increased LST in urban forests. Strong interactions between elevation and dominant species were generally observed in both hot and cool spots, but varied during different years. Although our field survey and remote-sensing data are from Xiamen City, our research method and results can be used as a reference for the investigation of urban spatial patterns in other cities.

\section{Acknowledgments}

This work was supported by National Science Foundation of China (31470578, 41301484, 41471366 and 31200363), National Forestry Public Welfare Foundation of China (201304205), Fujian Provincial Department of S\&T Project (2013YZ0001-1, 2016Y0083, 2014J05044 and 2015Y0083), Xiamen Municipal Department of Science and Technology (3502Z20130037 and 3502Z20142016), and Youth Innovation Promotion Association CAS (2014267). We are grateful to Professor Li Hu for his helpful suggestions.

\section{Appendix A. Supplementary data}

Supplementary data related to this article can be found at http:// dx.doi.org/10.1016/j.envpol.2016.06.004.

\section{References}

Ahmad, S.S., Aziz, N., Butt, A. et al., 2015. Spatio-temporal surveillance of wate based infectious disease (malaria) in Rawalpindi, Pakistan using geostatistical modeling techniques. Environ. Monit. Assess. 187, 1-15.

Barrell, J., Grant, J., 2013. Detecting hot and cold spots in a seagrass landscape using local indicators of spatial association. Landsc. Ecol. 28, 2005-2018.

Bump, J.K., Peterson, R.O., Vucetich, J.A., 2009. Wolves modulate soil nutrient heterogeneity and foliar nitrogen by configuring the distribution of ungulate carcasses. Ecology 90, 3159-3167.

Buyantuyev, A., Wu, J., 2010. Urban heat islands and landscape heterogeneity: linking spatiotemporal variations in surface temperatures to land-cover and socioeconomic patterns. Landsc. Ecol. 25, 17-33.

Cañadas, E.M., Fenu, G., Peñas, J., et al., 2014. Hotspots within hotspots: endemic plant richness, environmental drivers, and implications for conservation. Biol. Conserv. 170, 282-291.

Cetin, M., 2015. Using GIS analysis to assess urban green space in terms of accessibility: case study in Kutahya. Int. J. Sustain. Dev. World Ecol. 22, 420-424.

Chen, X.L., Zhao, H.M., Li, P.X., et al., 2006. Remote sensing image-based analysis of the relationship between urban heat island and land use/cover changes. Remote Sens. Environ. 104, 133-146.

Connors, J.P., Galletti, C.S., Chow, W.T.L., 2013. Landscape configuration and urban heat island effects: assessing the relationship between landscape characteristics and land surface temperature in Phoenix, Arizona. Landsc. Ecol. 28, 271-283.

Grassein, F., Lavorel, S., Till-Bottraud, I., 2014. The importance of biotic interactions and local adaptation for plant response to environmental changes: field evidence along an elevational gradient. Glob. Change Biol. 20, 1452-1460.

Heffernan, J.B., Soranno, P.A., Angilletta, M.J., et al., 2014. Macrosystems ecology: understanding ecological patterns and processes at continental scales. Front Ecol. Environ. 12, 5-14.

Ivajnšič, D., Kaligarič, M., Žiberna, I., 2014. Geographically weighted regression of the urban heat island of a small city. Appl. Geogr. 53, 341-353.

Josefsson, T., Hornberg, G., Ostlund, L., 2009. Long-term human impact and vegetation changes in a boreal forest reserve: implications for the use of protected areas as ecological references. Ecosystems 12, 1017-1036.

Kong, F., Yin, H., James, P., et al., 2014. Effects of spatial pattern of greenspace on urban cooling in a large metropolitan area of eastern China. Landsc. Urban Plan. $128,35-47$

Kuang, W., Liu, Y., Dou, Y., et al., 2014. What are hot and what are not in an urban landscape: quantifying and explaining the land surface temperature pattern in 
Beijing, China. Landsc. Ecol. 30, 1-17.

Levy, O., Ball, B.A., Bond-Lamberty, B., et al., 2014. Approaches to advance scientific understanding of macrosystems ecology. Front. Ecol. Environ. 12, 15-23.

Li, J., Song, C., Cao, L., et al., 2011. Impacts of landscape structure on surface urban heat islands: a case study of Shanghai, China. Remote Sens. Environ. 115 3249-3261.

Lin, W., Yu, T., Chang, X., Wu, W., Zhang, Y., 2015. Calculating cooling extents of green parks using remote sensing: method and test. Landsc. Urban Plan. 134, $66-75$.

Long, J.R.D., Kardol, P., Sundqvist, M.K., et al., 2015. Plant growth response to direct and indirect temperature effects varies by vegetation type and elevation in subarctic tundra. Oikos 124, 772-783.

Mahboubi, P., Parkes, M., Stephen, C., et al., 2015. Using expert informed GIS to locate important marine social-ecological hotspots. J. Environ. Manag. 160, $342-352$.

Naidoo, G. Chirkoot, D., 2004. The effects of coal dust on photosynthetic performance of the mangrove, Avicennia marina in Richards Bay, South Africa. Environ. Pollut. 127, 359-366.

Norton, B.A., Coutts, A.M., Livesley, S.J., et al., 2015. Planning for cooler cities: a framework to prioritise green infrastructure to mitigate high temperatures in urban landscapes. Landsc. Urban Plan. 134, 127-138.

Ord, J.L., Getis, A., 1995. Local spatial autocorrelation statistics: distributional issues and an application. Geogr. Anal. 27, 286-306.

Peeters, A., Zude, M., Käthner, J., et al., 2015. Getis-Ord's hot- and cold-spot statistics as a basis for multivariate spatial clustering of orchard tree data. Comput. Electron. Agric. 111, 140-150.

Ren, Y., Deng, L., Zuo, S., et al., 2014. Geographical modeling of spatial interaction between human activity and forest connectivity in an urban landscape of southeast China. Landsc. Ecol. 29, 1741-1758.

Salvati, L., Ranalli, F., Gitas, I., 2014. Landscape fragmentation and the agro-forest ecosystem along a rural-to-urban gradient: an exploratory study. Int. J.
Sustain. Dev. World Ecol. 21, 160-167.

Shoshany, M., Goldshlager, N., Kutiel, P., Grossman, D., 2005. Chapter 9.Man-Landscape relationships in mediterranean areas: a study of landscape changes in the mount carmel. In: Recent Dynamics of the Mediterranean Vegetation and Landscape. Wiley Online Library, pp. 95-104. http://onlinelibrary.wiley.com/ doi/10.1002/0470093714.ch9/summary.

Tayyebi, A., Jenerette, G.D., 2016. Increases in the climate change adaption effectiveness and availability of vegetation across a coastal to desert climate gradient in metropolitan Los Angeles, CA, USA. Sci. Total Environ. 548-549, 60-71.

Van Heerden, P.D.R., Kruger, G.H.J., Louw, M.K., 2007. Dynamic responses of photosystem II in the Namib Desert shrub, Zygophyllum prismatocarpum, during and after foliar deposition of limestone dust. Environ. Pollut. 146, 34-45.

Wang, J.F., Hu, Y., 2012. Environmental health risk detection with GeogDetector. Environ. Model. Softw. 33, 114-115.

Wang, J., Zhang, T., Fu, B., 2016. A measure of spatial stratified heterogeneity. Ecol. Indic. 67, 250-256.

Weng, J.H., Lu, D., Schubring, J., 2004. Estimation of land surface temperature-vegetation abundance relationship for urban heat island studies. Remote Sens. Environ. 89, 467-483.

Yuan, F. Bauer, M.E., 2007. Comparison of impervious surface area and normalized difference vegetation index as indicators of surface urban heat island effects in Landsat imagery. Remote Sens. Environ. 106, 375-386.

Zhao, J. Liu, X., Dong, R., 2016a. Landsenses ecology and ecological planning towards sustainable development. Int. J. Sustain. Dev. World Ecol. 293-297. http://dx.doi.org/10.1080/13504509.2015.1119215.

Zhao, S., Zhou, D., Liu, S., 2016b. Data concurrency is required for estimating urban heat island intensity. Environ. Pollut. 208, 118-124.

Zhao, X.F., Huang, J.C., Ye, H., et al., 2010. Spatiotemporal changes of the urban heat island of a coastal city in the context of urbanization. Int. J. Sustain. Dev. World Ecol. 17, 311-316. 